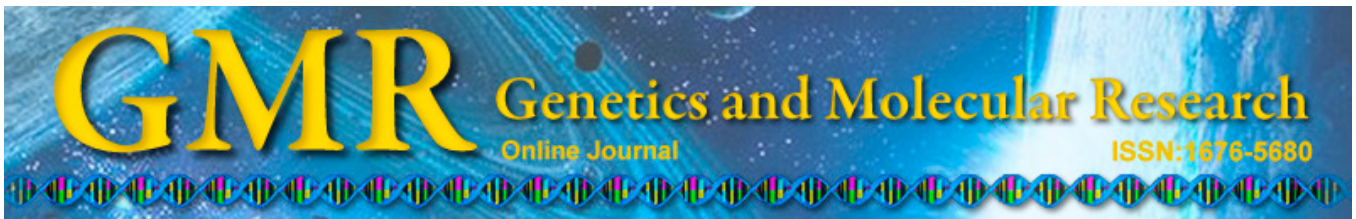

\title{
In silico analysis of gene content in tomato genomic regions mapped to the $T y-2$ resistance gene
}

\author{
Y.F. Liu, H.J. Wan, Y.P. Wei, R.Q. Wang, M.Y. Ruan, Q.J. Ye, Z.M. Li, \\ G.Z. Zhou, Z.P. Yao and Y.J. Yang
}

State key Laboratory Breeding Base for Zhejiang Sustainable Pest and Disease Control, Institute of Vegetables, Zhejiang Academy of Agricultural Sciences, Hangzhou, China

Corresponding author: H.J. Wan

E-mail: wanhongjian@sina.com

Genet. Mol. Res. 14 (3): 7947-7956 (2015)

Received August 15, 2014

Accepted March 18, 2015

Published July 17, 2015

DOI http://dx.doi.org/10.4238/2015.July.17.2

\begin{abstract}
Tomato yellow leaf curl virus is one of the main diseases affecting tomato production worldwide. Previous studies have shown that Ty-2 is an important resistance gene located between molecular markers C2_At2g28250 $(82.3 \mathrm{cM})$ and T0302 $(89.0 \mathrm{cM})$, and exhibits strong resistance to tomato yellow leaf curl virus in Asia. In this study, Ty-2 candidate genes were subjected to bioinformatic analysis for the sequenced tomato genome. We identified 69 genes between molecular markers C2_At2g28250 and T0302, 22 of which were disease-related resistant genes, including nucleotide binding site-leucine-rich repeat disease resistance genes, protease genes (protein kinase, kinase receptor, and protein isomerase), cytochromes, and transcription factors. Expressed sequence tag analysis revealed that $77.3 \%$ (17/22) of candidate disease-resistance genes were expressed, involving 143 expressed sequence tags. Based on full-length cDNA sequence analysis, 7 candidate genes were found, 4 of which were involved in tomato responses to pathogens. Microarray ex-
\end{abstract}


pression analysis also showed that most candidate genes were involved in the tomato responses to multiple pathogens, including fungi, viruses, and bacteria. RNA-seq expression analysis revealed that all candidate genes participated in tomato growth and development.

Key words: Disease resistance; Expressed sequence tag; Tomato; Ty-2; Tomato yellow leaf curl virus

\section{INTRODUCTION}

Tomato (Solanum lycopersicum L.) belongs to the family Solanaceae and is an important vegetable crop worldwide. Tomato yellow leaf curl virus (TYLCV) has severely affected the growth of tomato crops and world tomato production in recent years, and has been reported on a global scale (Accotto et al., 2000, 2003; Delatte et al., 2005; Wu et al., 2006; Lefeuvre et al., 2010; Melzer et al., 2010). Currently, the disease is primarily managed by spraying pesticides to control the virus vector whitefly. However, previous studies have shown that overuse of insecticides can increase the drug resistance of whitefly populations (Palumbo et al., 2001).

Breeding disease-resistant varieties is the most economic and efficient method for controlling TYLCV in tomato. However, no TYLCV-resistant gene resources exist in cultivated tomato cultivars, and wild tomato species such as Solanum pimpinellifolium, S. peruvianum, S. chilense, S. habrochaites, and S. cheesmaniae exhibit a high degree of disease resistance (Picó et al., 1996; Ji et al., 2007b; Scott, 2007; Vidavski, 2007). A total of 6 tomato TYLCV genes have been identified and named as Ty-1, Ty-2, Ty-3, Ty-4, Ty-5, and Ty-5 (Hanson et al., 2000, 2006; Garcia et al., 2007b; Ji et al., 2007a, 2009b; Hutton et al., 2012). These diseaseresistant genes were derived from different wild-type tomatoes. The $T y-2$ gene has been shown to exhibit clear resistance to TYLCV in India, Taiwan, Japan, and other Asian regions (Hanson et al., 2000), and has been widely used for TYLCV-resistance breeding in tomato. The $T y-2$ gene was first identified and located on the long arm of chromosome 11 between the molecular markers TG36 (84 cM) and TG393 (103 cM) (Hanson et al., 2000). Further studies localized this gene to between molecular markers TG36 $(84 \mathrm{cM})$ and TG26 $(92 \mathrm{cM})($ Hanson et al., 2006), and finally between C2_At2g28250 (82.3 cM) and T0302 (89.0 cM) (Ji et al., 2009a). Studies also reported that the marker TG105A was tightly linked to $T y-2$, but may also be related to the $I 2$ gene, which can cause false-positive results (Garcia et al., 2007a). Currently, though molecular markers for the $T y$-2 gene have been developed (Hanson et al., 2006; Garcia et al., 2007b; Ji et al., 2007a, 2009b; Hutton et al., 2012), further analysis on Ty-2 candidate genes has not been reported. In 2011, whole-genome sequencing of the tomato was completed (Tomato Genome Consortium, 2012), providing an opportunity for the screening of $T y-2$ candidate genes using a bioinformatic approach.

Based on the whole-genome sequence of tomato, $T y-2$ candidate genes were identified in silico in this study. Furthermore, the expression patterns of these genes were analyzed using expressed sequence tags (ESTs), full-length cDNA, microarray, and RNA-Seq. The results showed that $T y$ - 2 disease-resistant genes were restricted to within 22 candidate genes, providing a foundation for the further development of molecular markers for fine mapping and function analysis. In addition, these candidate genes were found to not only be involved in the growth and development of tomato, but they also participated in tomato responses to pathogens. 


\section{MATERIAL AND METHODS}

\section{Identification of $\boldsymbol{T y}-\mathbf{2}$ candidate genes}

According to a previous study, the $T y$ - 2 disease resistance gene is located between molecular markers C2_At2g28250 (82.3 cM) and T0302 (89.0 cM) (Ji et al., 2009a). In our study, based on the whole tomato genomic sequencing, the positions of these 2 molecular markers in the tomato genome database were 51305217 and 51879030, respectively. The $T y-2$ molecular marker gene number, position, and nucleotide sequence were retrieved using the Solanaceae genome website (http://solgenomics.net).

\section{EST-based expression analysis of candidate genes in the $T y$-2 regions}

Three EST databases (SOLHA, SOLLC, and SOLPN) were downloaded from the Solanaceae EST database website (http://biosrv.cab.unina.it/solestdb/). The local database was set up using the BioEdit software (Ibis Biosciences, Carlsbad, CA, USA). The Ty-2 candidate genes were compared to search for the corresponding candidate genes. The sequence similarity score was greater than 150 and the $\mathrm{E}$ value was less than -30 (Anderson and Brass, 1998).

\section{cDNA-based full-length expression analysis}

The full-length cDNAs of the $T y-2$ candidate genes in tomato were searched using Blast on the tomato full-length cDNAs website (http://www.pgb.kazusa.or.jp/kaftom/) with the selected $\mathrm{E}$ value of -30 .

\section{Microarray- and RNA-Seq-based expression analysis of $T y$-2 candidate genes}

The microarray analysis platform included the tomato TOM2 database. Tomato $T y-2$ candidate gene array probes were retrieved using Blast (http://ted.bti.cornell.edu/cgi-bin/TFGD/ array/blast.cgi). For genes with multiple corresponding probes, the gene probe with the highest E-value was selected. The RNA-Seq analysis platform was the tomato tissue expression dataset DOO4. The software $\mathrm{MeV}$ was used for clustering analysis of expression data to conduct.

\section{RESULTS}

\section{Identification of $T y$ - 2 candidate genes in tomato}

Previous studies showed that $T y-2$ disease-resistant genes were located between molecular markers C2_At2g28250 (82.3 cM) and T0302 (89.0 cM) (Ji et al., 2009a). Based on the tomato genome sequence, the physical distance between these 2 molecular markers was $573,813 \mathrm{bp}$. Sixty-nine genes were annotated in this region according to the analysis of the tomato genome sequence, 22 of which were related to resistance to TYLCV disease based on previous transcriptomics studies (Chen et al., 2013) (Table 1). These disease-resistance genes were divided into 4 classes, including nucleotide binding site-leucine-rich repeat (NBSLRR), protease genes (protein kinase, kinase receptor, and protein isomerase), cytochromes, and transcription factors. Among the 22 candidate genes, 5 belonged to the NBS-LRR family, 
3 were receptor-like protein kinase family members, 3 were transcription factors, 2 were protein isomerases, 2 were involved in oxidation reduction in organisms, 1 encoded structural proteins, 1 encoded allergic pathogenic proteins, 2 were ubiquitin ligases, and 1 was related to cytochromes.

Table 1. $T y$-2 candidate genes.

\begin{tabular}{lcccl}
\hline Gene Name & Physical position (bp) & Size (bp) & Exon No. & Predicted protein function \\
\hline Solyc11g069590.1.1 & $51305442-51307901$ & 2460 & 6 & Receptor-like protein kinase \\
Solyc1lg069620.1.1 & $51346017-51349831$ & 3815 & 2 & CC-NBS-LRR, resistance protein \\
Solyc11g069630.1.1 & $51353722-51351654$ & 2069 & 2 & Receptor-like protein kinase \\
Solyc11g069660.1.1 & $51374081-51371454$ & 2628 & 1 & NBS-LRR, resistance protein \\
Solyc11g069670.1.1 & $51376047-51375871$ & 177 & 1 & Disease resistance protein R3a-like protein \\
Solyc11g069690.1.1 & $51383609-51389361$ & 5753 & 4 & Protein disulfide isomerase \\
Solyc11g069770.1.1 & $51482195-51486514$ & 4320 & 2 & Transcription factor MADS-box protein \\
Solyc11g069800.1.1 & $51514060-51512528$ & 1533 & 1 & Cytochrome P450 \\
Solyc11g069860.1.1 & $51566625-51571040$ & 4416 & 4 & Glutaredoxin \\
Solyc11g069920.1.1 & $51610237-51610882$ & 646 & 2 & NBS, resistance protein fragment \\
Solyc11g069930.1.1 & $51612577-51616365$ & 3789 & 2 & Disease resistance protein R3a-like protein \\
Solyc11g069940.1.1 & $51622746-51623174$ & 429 & 1 & Glutaredoxin \\
Solyc11g069960.1.1 & $51646669-51643713$ & 2957 & 3 & Receptor like kinase, RLK \\
Solyc11g069970.1.1 & $51650118-51649333$ & 786 & 1 & Harpin-induced 1 \\
Solyc11g069990.1.1 & $51664081-51661763$ & 2319 & 1 & NBS-LRR, resistance protein \\
Solyc11g070000.1.1 & $51664969-51664166$ & 804 & 1 & NBS, resistance protein fragment \\
Solyc11g070040.1.1 & $51683590-51685026$ & 1437 & 1 & Pentatricopeptide repeat-containing protein \\
Solyc11g070070.1.1 & $51703065-51701395$ & 1671 & 2 & Zinc finger CCCH domain-containing protein 39 \\
Solyc11g071190.1.1 & $51800380-51801390$ & 1011 & 2 & C3HC4 type zinc-finger domain-containing protein \\
Solyc11g071230.1.1 & $51854001-51851248$ & 2754 & 3 & Galactosylgalactosylxylosyl protein 3-beta-glucuronosyl transferase \\
Solyc11g071240.1.1 & $51857580-51859206$ & 1627 & 2 & U-box domain-containing protein \\
Solyc11g071260.1.1 & $51882669-51878146$ & 4524 & 5 & Ubiquitin-conjugating enzyme E2 \\
\hline
\end{tabular}

According to the gene clusters and tandem repeats criteria used in previous studies (Meyers et al., 2003; Yang et al., 2008; Huang et al., 2012), further analysis revealed that the 5 NBS-LRR family genes in the $T y$-2 disease-resistant genes region belonged to 2 gene clusters. Solyc11g069620.1.1 and Solyc11g069660.1.1 belonged to the same gene cluster, and exhibited tandem repeat modes; the other 3 NBS-LRR family genes (Solyc11g069920.1.1, Solyc11g069990.1.1, and Solyc11g070000.1.1) belonged to another cluster.

\section{EST expression analysis}

To determine the potential function of $T y-2$ candidate genes, the expression of the 22 candidate genes was analyzed using the tomato EST database (http://biosrv.cab.unina.it/ solestdb/). The results showed that 17 candidate genes were supported by 143 ESTs (Table 2). Each gene harbored various numbers of ESTs, ranging from 1-25. These ESTs were mostly from cultivated tomato (S. lycopersicum), but some were also identified in wild-type tomatoes, in which the similarity between the $T y$-2 candidate gene Solyc11g069590.1.1 and EST (AW618332) from S. pennellii was as high as $99 \%$.

According to previously reported criteria, 3 different categories of genes were identified based on the number of EST matches to a gene. If the number of EST matches to a gene were $<7$, it was considered minimally expressed; if matches were between 7 and 200, it was considered relatively highly expressed; and if matches exceeded 200, it was considered highly expressed (Mahalingam et al., 2003; Dubey and Chandel, 2010). Among the 17 candidate genes, 9 showed relatively high expression levels, including Solyc11g069590.1.1, 
Solyc11g069620.1.1, Solyc11g069660.1.1, Solyc11g069800.1.1, Solyc11g069860.1.1, Solyc11g069940.1.1, Solyc11g069990.1.1, Solyc11g070000.1.1, and Solyc11g071260.1.1. Of these 9, the EST matches to Solyc11g069800.1.1 reached a maximum of 24. The remaining 8 genes were minimally expressed, with EST matches of $<7$, respectively. No highly expressed genes were observed.

Table 2. EST-based expression analysis of $T y$-2 candidates.

\begin{tabular}{lccc}
\hline Genes & \multicolumn{2}{c}{ EST database } \\
\cline { 2 - 4 } & SOLHA & SOLLC & SOLPN \\
\hline Solyc11g069590.1.1 & 0 & 9 & 1 \\
Solyc11g069620.1.1 & 0 & 20 & 0 \\
Solyc11g069660.1.1 & 0 & 14 & 0 \\
Solyc11g069670.1.1 & 0 & 5 & 0 \\
Solyc11g069690.1.1 & 0 & 2 & 0 \\
Solyc11g069800.1.1 & 1 & 9 & 0 \\
Solyc11g069860.1.1 & 0 & 4 & 0 \\
Solyc11g069930.1.1 & 0 & 13 & 0 \\
Solyc11g069940.1.1 & 0 & 3 & 0 \\
Solyc11g069960.1.1 & 0 & 9 & 0 \\
Solyc11g069990.1.1 & 0 & 7 & 0 \\
Solyc11g070000.1.1 & 0 & 1 & 0 \\
Solyc11g070040.1.1 & 0 & 1 & 0 \\
Solyc11g070070.1.1 & 0 & 2 & 0 \\
Solyc11g071230.1.1 & 0 & 1 & 0 \\
Solyc11g071240.1.1 & 0 & 17 & 0 \\
Solyc11g071260.1.1 & 0 & 24 & 0 \\
\hline
\end{tabular}

SOLHA: Solanum habrochaites; SOLLC: S. lycopersicum L; SOLPN: S. pennellii.

\section{Full-length cDNA expression analysis}

Full-length cDNA sequences of the 22 tomato $T y$ - 2 candidate genes were retrieved using the online Blast software from the tomato full-length cDNA database website (http://www. pgb.kazusa.or.jp/kaftom/). One or more full-length cDNAs were found for 7 of the $22 T y-2$ candidate genes (Table 3). The functions of these full-length cDNAs included tomato growth and development and different pathogen stress treatment. For example, Solyc11g069590.1.1 participates in the growth and development of fruits, and Solyc11g069860.1.1 and Solyc11g071260.1.1 participate in the maturing process of fruit. In contrast, the remaining 4 genes were involved in the tomato responses to pathogens.

Table 3. cDNA-based full-length expression analysis.

\begin{tabular}{|c|c|c|c|c|c|c|}
\hline Genes & cDNA & Description & Identity (\%) & Alignment length & E value & Bit Score \\
\hline Solyc11g069590.1.1 & LEFL2012B21 & Stage of fruit development & 99 & 1464 & 0 & 2894 \\
\hline Solyc11g069800.1.1 & LEFL1089CF06 & Pathogen-treated (Leaf) & 99 & 1533 & 0 & 3015 \\
\hline \multirow[t]{2}{*}{ Solyc11g069860.1.1 } & FC11AD08 & Maturing fruits & 99 & 432 & 0 & 825 \\
\hline & LEFL2002BC12 & Stage of fruit development & 99 & 432 & 0 & 811 \\
\hline Solyc11g069940.1.1 & LEFL1009CB09 & Pathogen-treated (Leaf) & 99 & 429 & 0 & 842 \\
\hline Solyc11g069960.1.1 & LEFL1020AF06 & Pathogen-treated (Leaf) & 99 & 1830 & 0 & 3510 \\
\hline \multirow[t]{2}{*}{ Solyc11g071230.1.1 } & LEFL1019AA04 & Pathogen-treated (Leaf) & 99 & 1335 & 0 & 2639 \\
\hline & LEFL1011BF06 & Pathogen-treated (Leaf) & 84 & 618 & $1.00 \mathrm{E}-120$ & 432 \\
\hline Solyc11g071260.1.1 & FC03AE04 & Maturing fruits & 99 & 474 & 0 & 924 \\
\hline
\end{tabular}




\section{Microarray expression analysis of $T y-2$ candidate genes}

The expression patterns of the candidate genes were further analyzed using tomato TOM2 array data. Twelve candidate genes were found using corresponding probes. Under tomato spotted wilt virus infection, the expression levels of Solyc11g069990.1.1 and Solyc11g069660.1.1 in the root were high, while expression of the other genes was low. Except for 3 candidate genes (Solyc11g069590.1.1, Solyc11g069960.1.1, and Solyc11g069990.1.1), the expression levels of most genes in the leaves were high (Figure 1). Under the arbuscular mycorrhizal fungus Glomus mosseae infection, the expression levels of Solyc11g069990.1.1 and Solyc11g069800.1.1 in the roots were relatively high, while the expression of the other genes was low. In the leaves, 2 candidate genes (Solyc11g069590.1.1 and Solyc11g069770.1.1) were highly expressed, 4 genes (Solyc11g069620.1.1, Solyc11g069670.1.1, Solyc11g069930.1.1, and Solyc11g070000.1.1) were moderately expressed, and the expression levels of the remaining genes were low.

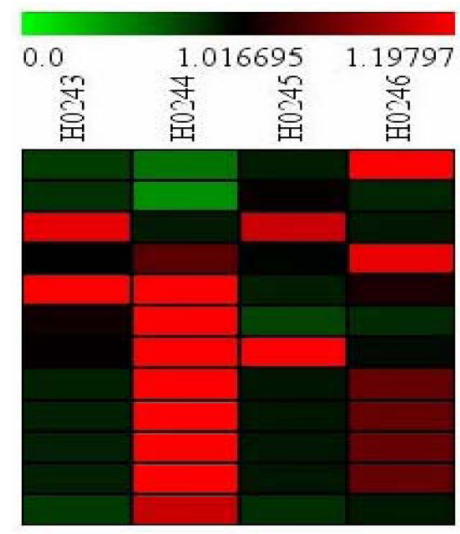
Solyc11g069590.1.1
Solyc11 g069960.1.1
Solyc11 g069990.1.1
Solyc11g069770.1.1
Solyc11 g069660.1.1
Solyc11 1069940.1 .1
Solyc11g069800.1.1
Solyc11g069620.1.1
Solyc11g069670.1.1
Solyc11 g069930.1.1
Solyc11g070000.1.1
Solyc11g071260.1.1

Figure 1. Expression profiles of tomato $T y-2$ candidates based on tomato microarray. Expression based on TOM2 Array. H0243: the Moneymaker root relative expression in plants systemically infected with tomato spotted wilt virus at 51 days; H0244: the Moneymaker leaves relative expression in plants systemically infected with tomato spotted wilt virus at 51 days. H0245: the Moneymaker root relative expression in plants colonized by the arbuscular mycorrhizal fungus Glomus mosseae at 51 days; H0246: the Moneymaker leaves relative expression in plants colonized by the arbuscular mycorrhizal fungus Glomus mosseae at 51 days.

\section{Expression analysis of $T y$-2 candidate genes based on RNA-Seq}

Based on the tomato RNA-Seq dataset, the expression patterns of the $22 T y-2$ candidate genes in different stages of vegetative and reproductive development were determined (Figure 2). The results showed that the expression levels of Solyc11g069940.1.1, Solyc11g069930.1.1, Solyc11g069800.1.1, Solyc11g069860.1.1, Solyc11g069690.1.1, and Solyc11g071260.1.1 were high in all tested tissues; the expression level of Solyc11g069990.1.1 was moderate in all tested tissues; and the expression levels of Solyc11g071230.1.1, Solyc11g070070.1.1, Solyc11g071240.1.1, and Solyc11g069660.1.1 were moderate in most tissues tested. In addition, compared with those in the root, leaf, flower, and bud, the expression level of Solyc11g069900.1.1 was higher during fruit development. The expression levels of Solyc11g069770.1.1 were moderate in the root, bud, and flower, while in other tissues, expression levels were low. The expression levels of the remaining genes were low in all tissues tested. 


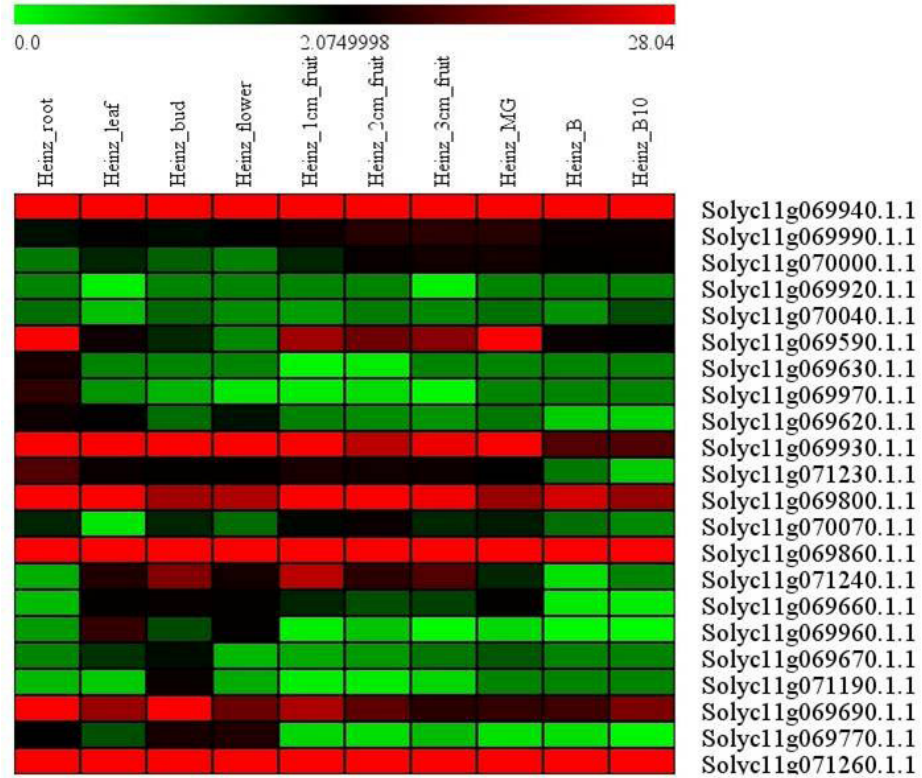

Figure 2. Expression profiles of tomato $T y-2$ candidate genes based on RNA-Seq data. The tissues from the left to right are roots, leaves, flower buds, fully opened flowers, and 1-cm, 2-cm, 3-cm, mature green, breaker, and breaker +10 fruits of tomato cultivar Heinz.

\section{DISCUSSION}

Currently, at least 6 TYLCV resistant genes (Ty-1,Ty-2, Ty-3, Ty-4, Ty-5, and Ty- 6 ) have been identified in tomato, and molecular markers and gene mapping of these resistance genes were analyzed in previous studies (Hanson et al., 2006; Garcia et al., 2007b; Ji et al., 2007a, 2009b; Hutton et al., 2012). Of them, Ty-1 and Ty-3 resistance genes have been cloned and found to encode the DFDGD motif, which belongs to the RNA-dependent RNA $\gamma$ polymerase family (Verlaan et al., 2013). However, cloning of the remaining TYLCV resistance genes has not been reported. In the present study, based on the mapping of $T y-2$ by previous researchers, candidate genes of $7 y-2$ were analyzed using bioinformatics and tomato wholegenome sequencing information (Tomato Genome Consortium, 2012) to facilitate the identification and cloning of the $T y-2$ resistance gene.

Our results revealed abundant disease-resistant candidate genes in the chromosome regions encompassing the tomato TYLCV resistance locus $T y-2$ (Table 1). These disease-resistance genes accounted for $31.9 \%$ (22/69) of the total gene number in the $T y-2$ region, and for $0.92 \%(22 / 2385)$ of genes in tomato chromosome 11 . Among the 22 candidate genes, 5 encoded NBS domain proteins and belonged to the NBS-LRR family of resistance genes. In all cloned disease-resistant genes in plants, most genes belonged to the NBS-LRR family (Liu et al., 2007). In tomato, most cloned resistance genes also belong to this family, including Prf, I2, Mi-1, Mi-9, Sw-5/Mi, and Hero (Salmeron et al., 1996; Ori et al., 1997; Brommonschenkel et al., 2000; Ernst et al., 2002; Tameling et al., 2002; Jablonska et al., 2007). The receptor-like protein kinase is also considered as a disease resistance gene family, and these genes have been shown to be involved in plant defense against pathogens (Kruijt et al., 2005; Hok et al., 2011). 
Previous studies showed that the wheat rust resistance gene $\operatorname{Lr} 10$ and rice blast resistance gene $P i-d 2$ both encoded receptor-like protein kinases (Feuillet et al., 1997; Kouzai et al., 2013). In this study, the candidate genes Solyc11g069590.1.1 and Solyc11g069630.1.1 also encoded receptor-like protein kinase, indicating that the 2 candidate genes are associated with plant disease resistance. The pentatricopeptide repeat-containing protein is an RNA binding protein (Saha et al., 2007) that regulates mitochondrial and chloroplast genes through post-transcription modification of RNA. Expression analysis of the rice response to Xanthomonas oryzae showed that the gene encoding a pentatricopeptide repeatcontaining protein was upregulated (Zhou et al., 2010), suggesting that these genes are induced by pathogenic bacteria.

The full-length cDNA library and the EST database are important resources for gene expression analysis (Adams et al., 1995). In this study, these databases were used to analyze candidate $T y-2$ genes. EST-based expression analysis revealed that $77.3 \%(17 / 22)$ of candidate disease-resistant genes contained EST in the regions of the $T y-2$ gene, indicating that most $T y-2$ candidate genes were expressed in tomato. In addition, $53 \%(9 / 17)$ of expressed candidate genes contained more than 7 ESTs, indicating their relatively high expression levels (Mahalingam et al., 2003; Dubey and Chandel, 2010).

Based on RNA-Seq datasets, the expression patterns of the 22 tomato $T y-2$ candidate genes in the roots, leaves, blossom buds, other tissues as well as the fruit development stages were examined (Figure 2). The results showed high expression levels of 6 genes (Solyc11g069940.1.1, Solyc11g069930.1.1, Solyc11g069800.1.1, Solyc11g069860.1.1, Solyc11g069690.1.1, and Solyc11g071260.1.1) in all tested tissues. These 6 genes encoded redox protein, cytochrome, plant disease resistance proteins, isomerase, and ubiquitin conjugating enzyme, respectively. For the remaining 16 genes, each was expressed in at least 1 tissue under normal growth conditions. These results showed that the genes were not only involved in tomato responses to environment stresses, but they also participated in tomato growth and development.

\section{CONCLUSIONS}

Using bioinformatic methods, 22 disease-resistance candidate genes of $T y-2$ were identified. The EST, cDNA, microarray, and RNA-Seq data of expression pattern results confirmed the involvement of these genes in tomato growth and development, as well as responses to multiple stresses. This study provides a foundation for the further development of molecular markers for the fine mapping and function analysis of $T y-2$ resistance genes.

\section{ACKNOWLEDGMENTS}

Research partially supported by the Young Talent Training Program for Zhejiang Academy of Agricultural Technology Application Research Project for Zhejiang Province (\#2013C32G4010256); the General Program from the National Natural Science Foundation of China (\#31301774 and \#31071800); Breeding of Vegetable Varieties in Zhejiang Province (\#2009C02006-1); Technological System of Ordinary Vegetable Industry (\#CARS-25-G-16); Zhejiang Provincial Vegetable Industry Innovation Team (\#2009R50026); Zhejiang Provincial major Agricultural Science and Technology Projects of New Varieties Breeding (\#2012C12903); and Mol Breeding of Major Vegetable and Functional Genomics Research (\#2012AA1001). 


\section{REFERENCES}

Accotto GP, Navas-Castillo J, Noris E, Moriones E, et al. (2000). Typing of tomato yellow leaf curl viruses in Europe. Eur. J. Plant Pathol. 106: 179-186.

Accotto G, Bragaloni M, Luison D, Davino S, et al. (2003). First report of tomato yellow leaf curl virus (TYLCV) in Italy. Plant Pathol. 52: 799-799.

Adams MD, Kerlavage AR, Fleischmann RD, Fuldner RA, et al. (1995). Initial assessment of human gene diversity and expression patterns based upon 83 million nucleotides of cDNA sequence. Nature 377: 3-174.

Anderson I and Brass A (1998). Searching DNA databases for similarities to DNA sequences: when is a match significant? Bioinformatics 14: 349-356.

Brommonschenkel SH, Frary A, Frary A and Tanksley SD (2000). The broad-spectrum tospovirus resistance gene $S w-5$ of tomato is a homolog of the root-knot nematode resistance gene Mi. Mol. Plant Microbe Interact. 13: 1130-1138.

Chen TZ, Lv YD, Zhao TM, Li N, et al. (2013). Comparative transcriptome profiling of a resistant $v s$ susceptible tomato (Solanum lycopersicum) cultivar in response to infection by tomato yellow leaf curl virus. Plos One 8: e80816.

Delatte H, Holota H, Naze F, Peterschmitt M, et al. (2005). The presence of both recombinant and nonrecombinant strains of tomato yellow leaf curl virus on tomato in Reunion Island. Plant Pathol. 54: 262-262.

Dubey M and Chandel G (2010). In silico survey and characterization of resistance gene analogues (RGAs) in the genomic regions encompassing gall midge resistance genes Gm4 and Gm5 in rice (Oryza sativa L.). Plant Omics 3: 140-148.

Ernst K, Kumar A, Kriseleit D, Kloos DU, et al. (2002). The broad-spectrum potato cyst nematode resistance gene (Hero) from tomato is the only member of a large gene family of NBS-LRR genes with an unusual amino acid repeat in the LRR region. Plant J. 31: 127-136.

Feuillet C, Schachermayr G and Keller B (1997). Molecular cloning of a new receptor-like kinase gene encoded at the Lr10 disease resistance locus of wheat. Plant J. 11: 45-52.

Garcia BE, Graham E, Jensen KS, Hanson P, et al. (2007a). Co-dominant SCAR marker for detection of the begomovirusresistance Ty-2 locus derived from Solanum habrochaites in tomato germplasm. Tomato Genet. Cooper. 57: 21-24.

Garcia BE, Martin CT and Maxwell DP (2007b). Detection methods for the Ty-1 gene for resistance to begomoviruses on chromosome 6 of tomato. Available at http: //www. plantpath. wisc. edu/GeminivirusResistant-Tomatoes/Markers/ MAS-Protocols/IntroTy1.Pdf. Accessed 02-6-2015.

Hanson PM, Bernacchi D, Green S, Tanksley SD, et al. (2000). Mapping a wild tomato introgression associated with tomato yellow leaf curl virus resistance in a cultivated tomato line. J. Am. Soc. Hortic. Sci. 125: 15-20.

Hanson P, Green S and Kuo G (2006). Ty-2, a gene on chromosome 11 conditioning geminivirus resistance in tomato. Tomato Genet. Cooper. 56: 17-18.

Hok S, Danchin EG, Allasia V, Panabieres F, et al. (2011). An Arabidopsis (malectin-like) leucine-rich repeat receptorlike kinase contributes to downy mildew disease. Plant Cell. Environ. 34: 1944-1957.

Huang S, Gao Y, Liu J, Peng X, et al. (2012). Genome-wide analysis of WRKY transcription factors in Solanum lycopersicum. Mol. Genet. Genomics 6: 495-513.

Hutton SF, Scott JW and Schuster DJ (2012). Recessive resistance to tomato yellow leaf curl virus from the tomato cultivar Tyking is located in the same region as $T y-5$ on chromosome 4. HortScience 47: 324-327.

Jablonska B, Ammiraju JS, Bhattarai KK, Mantelin S, et al. (2007). The Mi-9 gene from Solanum arcanum conferring heat-stable resistance to root-knot nematodes is a homolog of Mi-1. Plant Physiol. 143: 1044-1054.

Ji Y, Schuster DJ and Scott JW (2007a). Ty-3, a begomovirus resistance locus near the tomato yellow leaf curl virus resistance locus Ty-1 on chromosome 6 of tomato. Mol. Breeding 20: 271-284.

Ji Y, Scott J, Hanson P, Graham E, et al. (2007b). Sources of resistance, inheritance, and location of genetic loci conferring resistance to members of the tomato-infecting begomoviruses. In: Tomato Yellow Leaf Curl Virus Disease. Springer, Netherlands, 343-362.

Ji Y, Scott JW and Schuster DJ (2009a). Toward fine mapping of the tomato yellow leaf curl virus resistance gene $T y$-2 on chromosome 11 of tomato. HortScience 44: 614-618.

Ji Y, Scott JW, Schuster DJ and Maxwell DP (2009b). Molecular mapping of Ty-4, a new tomato yellow leaf curl virus resistance locus on chromosome 3 of tomato. J. Am. Soc. Hortic. Sci. 134: 281-288.

Kouzai Y, Kaku H, Shibuya N, Minami E, et al. (2013). Expression of the chimeric receptor between the chitin elicitor receptor CEBiP and the receptor-like protein kinase Pi-d2 leads to enhanced responses to the chitin elicitor and disease resistance against Magnaporthe oryzae in rice. Plant Mol. Biol. 81: 287-295.

Kruijt M, DE Kock M and de Wit P (2005). Receptor-like proteins involved in plant disease resistance. Mol. Plant Pathol. 6: 85-97.

Lefeuvre P, Martin DP, Harkins G, Lemey P, et al. (2010). The spread of tomato yellow leaf curl virus from the middle 
east to the world. Plos Pathog. 6: e1001164.

Liu J, Liu X, Dai L and Wang G (2007). Recent progress in elucidating the structure, function and evolution of disease resistance genes in plants. J. Genet. Genomics 34: 765-776.

Mahalingam R, Gomez-Buitrago A, Eckardt N, Shah N, et al. (2003). Characterizing the stress/defense transcriptome of Arabidopsis. Genome Biol. 4: R20.

Melzer M, Ogata D, Fukuda S, Shimabuku R, et al. (2010). First report of Tomato yellow leaf curl virus in Hawaii. Plant Dis. 94: 641-641.

Meyers BC, Kozik A, Griego A, Kuang H, et al. (2003). Genome-wide analysis of NBS-LRR-encoding genes in Arabidopsis. Plant Cell 15: 809-834.

Ori N, Eshed Y, Paran I, Presting G, et al. (1997). The $I 2 C$ family from the wilt disease resistance locus $I 2$ belongs to the nucleotide binding, leucine-rich repeat superfamily of plant resistance genes. Plant Cell 9: 521-532.

Palumbo J, Horowitz A and Prabhaker N (2001). Insecticidal control and resistance management for Bemisia tabaci. Crop Protect. 20: 739-765.

Picó B, Díez MJ and Nuez F (1996). Viral diseases causing the greatest economic losses to the tomato crop. II. The Tomato yellow leaf curl virus - a review. Sci. Hortic-Amsterdam 67: 151-196.

Saha D, Prasad A and Srinivasan R (2007). Pentatricopeptide repeat proteins and their emerging roles in plants. Plant Physiol. Biochem. 45: 521-534.

Salmeron JM, Oldroyd GED, Rommens CMT, Scofield SR, et al. (1996). Tomato Prf Is a member of the leucine-rich repeat class of plant disease resistance genes and lies embedded within the Pto kinase gene cluster. Cell 86: 123-133.

Scott JW (2007). Breeding for resistance to viral pathogens. Genetics Improvement of Solanaceous Crops (Maharaj K. Razdan and Autar K. Mattoo). Science Publishers, Enfield, NH, USA, 2: 447-474.

Tameling WIL, Elzinga SDJ, Darmin PS, Vossen JH, et al. (2002). The tomato R gene products $I-2$ and $M i-1$ are functional ATP binding proteins with ATPase activity. Plant Cell 14: 2929-2939.

Tomato Genome Consortium TG (2012). The tomato genome sequence provides insights into fleshy fruit evolution. Nature 485: 635-641.

Verlaan MG, Hutton SF, Ibrahem RM, Kormelink R, et al. (2013). The tomato yellow leaf curl virus resistance genes Ty-1 and Ty-3 are allelic and code for DFDGD-class RNA-dependent RNA polymerases. PloS Genet. 9: e1003399.

Vidavski F (2007). Exploitation of resistance genes found in wild tomato species to produce resistant cultivars; Pile up of resistant genes. In: Tomato Yellow Leaf Curl Virus Disease. Springer, Netherlands, 363-372.

Wu J, Dai F and Zhou X (2006). First report of tomato yellow leaf curl virus in China. Plant Dis. 90: 1359-1359.

Yang S, Zhang X, Yue JX, Tian D, et al. (2008). Recent duplications dominate NBS-encoding gene expansion in two woody species. Mol. Genet. Genomics 280: 187-198.

Zhou YL, Xu MR, Zhao MF, Xie XW, et al. (2010). Genome-wide gene responses in a transgenic rice line carrying the maize resistance gene Rxol to the rice bacterial streak pathogen, Xanthomonas oryzae pv. oryzicola. BMC Genomics 11: 78 . 